Selection 2 (2001) 1-2, 1-15

Available online at http://www.akkrt.hu

\title{
What can Invasion Analyses Tell us about Evolution under Stochasticity in Finite Populations?
}

\author{
S. R. PROULX and T. DAY* \\ Department of Zoology, University of Toronto, Toronto, Ontario, Canada \\ (Received: 14 May 2001, \\ Accepted: 24 August 2001)
}

\begin{abstract}
The geometric mean of fitness is considered to be the main indicator of evolutionary change in stochastic models. However, this measure was initially derived for models with infinite population sizes, where the long-term evolutionary behavior can be described with certainty. In this paper we begin an exploration of the limitations and utility of this approach to evolution in finite populations and discuss alternate methods for predicting evolutionary dynamics. We reanalyze a model of lottery competition under environmental stochasticity by including population finiteness, and show that the geometric mean predictions do not always agree with those based on the fixation probability of rare alleles. Further, the fixation probability can be inserted into adaptive dynamics equations to derive the mean state of the population. We explore the effects of increasing population size on these conclusions through simulations. These simulations show that for small population sizes the fixation probability accurately predicts the course of evolution, but as population size becomes large the geometric mean predictions are upheld. The two approaches are reconciled because the time scale on which the fixation probability approach applies becomes very large as population size grows.
\end{abstract}

Keywords: Life history evolution, evolutionarily stable strategy

\section{Introduction}

As biologists we often attempt to predict the outcome of evolution as though it were a deterministic process, despite the fact that it is inherently stochastic. This may in part be due to a belief that the main effect of stochasticity is to introduce noise, and not to alter the direction of evolution. However, this deterministic outlook is often retained even when stochasticity is believed to have a directional effect on evolution, and it is exemplified by studies which use geometric mean fitness to predict the course of evolution under environmental stochasticity (Gillespie, 1973; Seger and Brock-

* Corresponding author: Troy Day, Department of Zoology, University of Toronto, 25 Harbord St., Toronto, Ontario, Canada M5S 3G5, E-mail: proulx@proulxresearch.org mann, 1987). The idea is that, if the environment as a whole fluctuates from year to year, then after a series of new mutations and successive allelic replacements, the allele coding for the strategy with the highest geometric mean fitness will prevail [however, see Iwasa (1988)]. If the scenario under examination involves fitness interactions among individuals that result in density and/or frequencydependence, then typically such 'replacement criteria' become unworkable. In such cases one then uses the geometric mean fitness of a strategy when rare as a measure of its ability to invade the population (Turelli, 1978b; Ellner, 1996; Benton and Grant, 2000). While this latter extension is not meant to imply that an invading strategy (i.e. allele) will necessarily replace the resident strategy, such 'invasion criteria' can (and have) been used to find uninvadible strategies under environmental stochasticity (Turelli, 1978b; Real and Ellner, 
1992; Iwasa and Levin, 1995; Kisdi and Meszena, 1995; Ellner, 1996; Grant, 1997; Benton and Grant, 1999; Benton and Grant, 2000; Ranta et al., 2000). In either case, the goal has been to reduce the predictions of the stochastic evolutionary model to a single, deterministic outcome based on the maximization of geometric mean fitness. Our purpose here is to show that the geometric mean fitness of invading strategies can give an incomplete picture of adaptive evolution in some situations stemming from two linked features; the infinite population size assumption and deterministic interpretation. We focus on evolution in finite populations and demonstrate that another fitness measure, the probability of fixation, provides additional and sometimes more suitable information than the geometric mean.

\section{Deterministic models}

To better understand the limitations of fitness measures in stochastic models it is helpful to first look at their use in deterministic models, such as simple optimality models. All such models assume that some fitness measure (e.g. lifetime reproductive output) is maximized by natural selection, and consequently these models are based (at least implicitly) on a replacement criterion. When such replacement criteria are valid they provide a very powerful tool for understanding evolution. Once the realities of density- and frequency-dependence are incorporated in deterministic models, however, such replacement criteria typically become intractable, as there is generally no longer an optimization principle (Metz et al., 1996b; Heino et al., 1998a, b; Kisdi, 1998). Analyses are then usually based upon an invasion criterion that characterizes a strategy's growth factor when rare [this approach is the basis of all game-theoretic modeling (Maynard Smith, 1982)].

The goal of an invasion analysis is to determine whether a rare mutant strategy can invade a resident strategy. This can be done by deriving an expression for the per capita growth factor of a mutant $\left(\frac{N i_{t+1}}{N i_{t}}\right)$, or the per capita change in frequency of a mutant $\left(\frac{p_{t+1}}{p_{t}}\right)$. To obtain the invasion criterion one takes the limit of this expression as the mutant frequency goes to zero. This limit (as frequency goes to zero) is the mathematical embodiment of rarity, and it is a central assumption in all invasion criteria. In mathematical language, it is a linearization of the joint dynamics of the mutant and resident strategies where the mutant strategy is absent, and it assumes that the mutant's growth factor when rare is determined by the environment that is set by the resident strategy. Figure 1a is a hypothetical curve for a mutant with a per capita growth factor of less than 1 at zero frequency. An evolutionarily stable strategy is one for which all

\section{Fig. 1a}

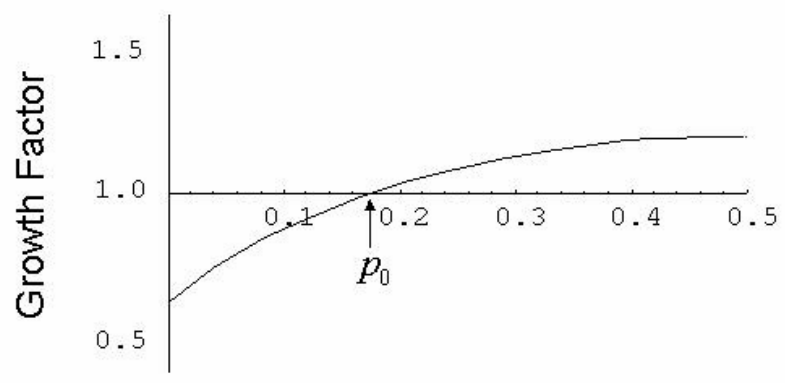

Mutant Frequency

Fig. 1b

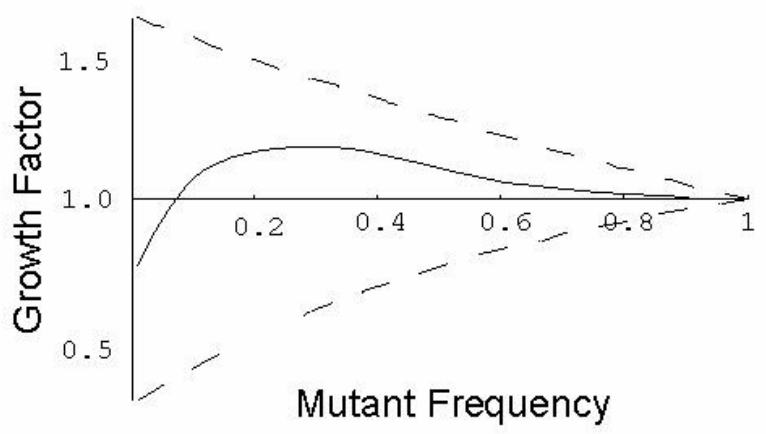

FIG. 1. Hypothetical plots of the mutant growth factor for deterministic and stochastic models. Panel $a$ is for a deterministic model with frequency dependence. The mutant has a growth factor of less than one when rare, indicating that the frequency of the mutant would decrease to zero. However, if the frequency of the mutant passes $p_{0}$, then the growth factor is greater than one, and the mutant would increase in frequency until it replaced the resident alleles. Panel $b$ shows the same scenario for a stochastic case. There are two types of years, one which results in high mutant growth factors (upper dashed curve), and one which results in low mutant growth factors (lower dashed curve). Even though the geometric mean growth factor of the mutant is less than one for low frequency, a run of good years would result in fixation of the mutant allele 
mutant strategies have growth factors less than one when rare.

Notice that the above analysis (i.e. linearization) tacitly relies on the continuous state space of the model when taking the limit as frequency goes to zero. Of course, this implies an assumption of an infinite population size since otherwise the smallest non-zero frequency possible would be $1 / N$, where $N$ is the population size. We are primarily interested in knowing whether or not population finiteness can qualitatively alter predictions about evolution. Consider an example in which a rare mutant strategy goes extinct deterministically, but if the mutant reaches a threshold frequency, $p_{0}$, then it spreads to fixation (Fig. 1a). Now suppose that $p_{0}<1 / N$, so that the rarest possible mutant in the population is nevertheless above this threshold frequency. This will occur if the real population size is small enough and/or the threshold is low. Then once a single mutant copy exists, it will spread to fixation despite the fact that the above invasion criterion predicts it to go extinct.

This example illustrates that invasion criteria can easily fail in deterministic models because they are based on the per capita growth factor of mutants when they have zero frequency, and any real mutant will first appear at frequency $1 / N$ in a finite population. Notice, however, that invasion criteria can be rescued (conceptually at least) provided we are willing to assume that the population size is large enough. More specifically, we can usually assume a large enough (but still finite) population so that the growth factor of a mutant strategy at frequency $1 / N$ has the same sign as the growth factor of a mutant strategy at frequency zero (Fig. 1a). In such a case, even though the real growth factor of a mutant will differ quantitatively from that predicted by the invasion criterion, the qualitative behavior of the two will nevertheless coincide. This means that invasion criteria will work properly provided that the population size is larger than some fixed threshold. As we will argue next, the exact same issues arise in stochastic models, and it is of interest to know the degree to which large population size rescues the invasion criteria in this setting.

\section{Stochastic models and the derivation of the geometric mean principle}

Geometric mean fitness is probably the most widely employed fitness measure under environmental stochasticity, and the geometric mean fitness of a mutant when rare has also been used as an invasion criterion in many studies (Turelli, 1978b; Real and Ellner, 1992; Kisdi and Meszena, 1995; Ranta et al., 2000). The reasoning behind using the geometric mean in stochastic models initially came from a stochastic model that predicted a 'deterministic' outcome (Gillespie, 1973). The original model is one of two strategies, each growing without density dependence or interference from the other strategy, and having its reproductive success in each year determined by the environment in that year (appendix $A$ ). In these models, even though the frequency of a mutant may go up or down in a particular generation, the frequency of the mutant becomes either virtually 0 or virtually 1 , depending on the geometric mean of relative fitness. The mathematical statement is that for any small neighborhood around 0 (or 1), the probability that the allele frequency is in that neighborhood approaches 1 as time goes to infinity (Gillespie, 1973). The strategy which becomes fixed is the strategy with the largest geometric mean fitness. These results have been interpreted as implying a geometric mean maximization principle, and thus the geometric mean has often been used as a replacement criterion.

Although the utility of the geometric mean was first shown for frequency and density independent processes, it was later applied as a heuristic tool to more complex processes, by using the geometric mean growth factor of a mutant strategy when rare as an invasion criterion (Turelli, 1978a; Chesson and Warner, 1981; Bulmer, 1985; Chesson, 1985). Although no proof was provided in these early studies, the logic was that such invasion criterion could be constructed for stochastic systems just as they are for deterministic systems. This logic was later upheld by rigorous mathematical treatments for a variety of models (Ellner, 1984; Chesson and Ellner, 1989; Ferriere and Gatto, 1995), and the idea that the geometric mean growth factor of a rare strategy can determine invasion success in any model has become prevalent in the theoretical lit- 
erature (Metz et al., 1992; Ellner, 1996; Benton and Grant, 2000). However, just as with the construction of deterministic invasion criteria, these treatments of stochastic invasion criteria consider only situations in which the state space of the model is continuous, and thus they include an infinite population size assumption.

Figure $1 \mathrm{~b}$ depicts a hypothetical example for a model of environmental stochasticity. The dashed curves depict two different types of year, while the solid curve is the geometric mean of the mutant growth factor. Because selection is highly variable, the new strategy has a low geometric mean growth factor when rare, and according to the invasion analysis this strategy will go extinct. But the dashed curves show that there is some possibility of increasing in frequency, and that this is true for any current frequency. Consequently, if the population is finite, and a run of years happens to favor the mutant, it may become fixed (alternatively, a run of bad years could drive it extinct). This uncertainty in the evolutionary outcome is often precluded in an infinite population when frequency is modeled as a continuous variable, because, under the linearization that is used in calculating the invasion measure, it takes an infinite number of steps for a rare allele to escape its infinitesimally small starting frequency. This ensures that the allele 'experiences' a perfectly representative collection of environmental states while it is still rare by forcing it to remain at zero frequency for an arbitrarily long period of time, and it is the reason why invasion criterion based on the geometric mean fitness when rare can accurately capture the invasion dynamics in models with a continuous state space. For a finite population, however, this will no longer be true because there is always some probability that the mutant allele escapes rarity before all possible environmental states are experienced.

In small populations stochastic effects due to drift alone will be large enough that predictions from infinite population models will not apply. The effect of stochasticity may be to alter the mean state, or to increase the variance of the system (Iwasa, 1988). This effect may be pronounced even for moderately sized populations, but of course it should tend towards zero as population size approaches infinity.

\section{The fixation probability as an indicator of evolutionary change}

To address the issues raised above about evolution in finite populations, another useful measure (rather than the geometric mean) is the fixation probability of a rare allele. The population genetics literature has relied heavily upon fixation probability [e.g. Crow and Kimura (1970)], and it has been used to infer the directionality of adaptive evolution (Gillespie, 1974; Otto and Whitlock, 1997; Proulx, 2000). If the fixation probability of a mutant allele is greater than neutral, then, in one sense, that allele is selectively favored.

A neutral allele in a haploid organism, i.e. an alternative allele which is different from the resident only in name (sequence) but not function, will become fixed with a probability of $1 / N$, when initially present in a single copy. Although one might guess that for a slightly favored allele, the fixation probability in a large population would be similar to the fixation probability of a neutral allele, this is not the case. An early study of Haldane showed that the fixation probability of a selectively favored allele is twice the selective advantage in an infinite population (Crow and Kimura, 1970). This result comes from a branching process approximation, where the mutant alleles are thought of as an independent population reproducing without density or frequency dependence. (Technically, the "fixation probability" is assumed to be one minus the extinction probability.) A mutant which is at a selective disadvantage is guaranteed to go extinct in the long run, and so has a fixation probability of 0 .

The same branching process approach can be taken for a model with environmental stochasticity, and the geometric mean fitness determines whether the fixation probability of a mutant allele is greater than 0 . In much the same way as for constant environments, an allele with a geometric mean greater than 1 has a positive fixation probability, whereas an allele with a geometric mean of less than 1 has a fixation probability of 0 in a very large population (Haccou and Iwasa, 1996). So, the geometric mean fitness of rare mutants describes the limit of the fixation probability as population size becomes large. However, it only partially distinguishes alleles which have fixation probabilities greater than neutral alleles and those which have fixation prob- 
abilities less than neutral alleles. In particular, an allele which has a geometric mean fitness of greater than 1 will have a greater than neutral fixation probability (in a large population), but an allele with a geometric mean fitness of less than 1 may still have a fixation probability greater than a neutral allele, even for very large population sizes. This situation is graphically depicted in Figure 2, which is motivated by the examples presented later in this paper.

Thus, in a small population, the geometric mean fitness may not indicate the magnitude of the fixation probability, and there are at least two reasons to expect that it is the fixation probability rather than the geometric mean that reveals important information for evolution in finite populations.

\subsection{Markovian 2-allele models}

The first reason comes from considering only two alleles at a time (which is a common assumption in many models that use invasion criteria) and asking how much time the system spends fixed for each allele. If we assume that mutations are rare compared to the time scale at which fixation occurs, and that the two alleles can mutate with the same probability, then the state of the population is a very simple Markov chain (see Iwasa [1988] for a similar approach). In any step, the population can either remain in its current state, or switch states. If we write the probability of allele $A$ fixing in a population of allele $B$ when rare as $U_{A, B}$, then the matrix describing the Markov chain is

$$
M=\left(\begin{array}{cc}
1-U_{B, A} & U_{B, A} \\
U_{A, B} & 1-U_{A, B}
\end{array}\right) .
$$

The proportion of time the population spends fixed for allele $A$ is given by $\frac{U_{A, B}}{U_{A, B}+U_{B, A}}$, which depends only on the relative fixation probabilities, but not on their magnitudes. Therefore, the allele which has the higher fixation probability is the one

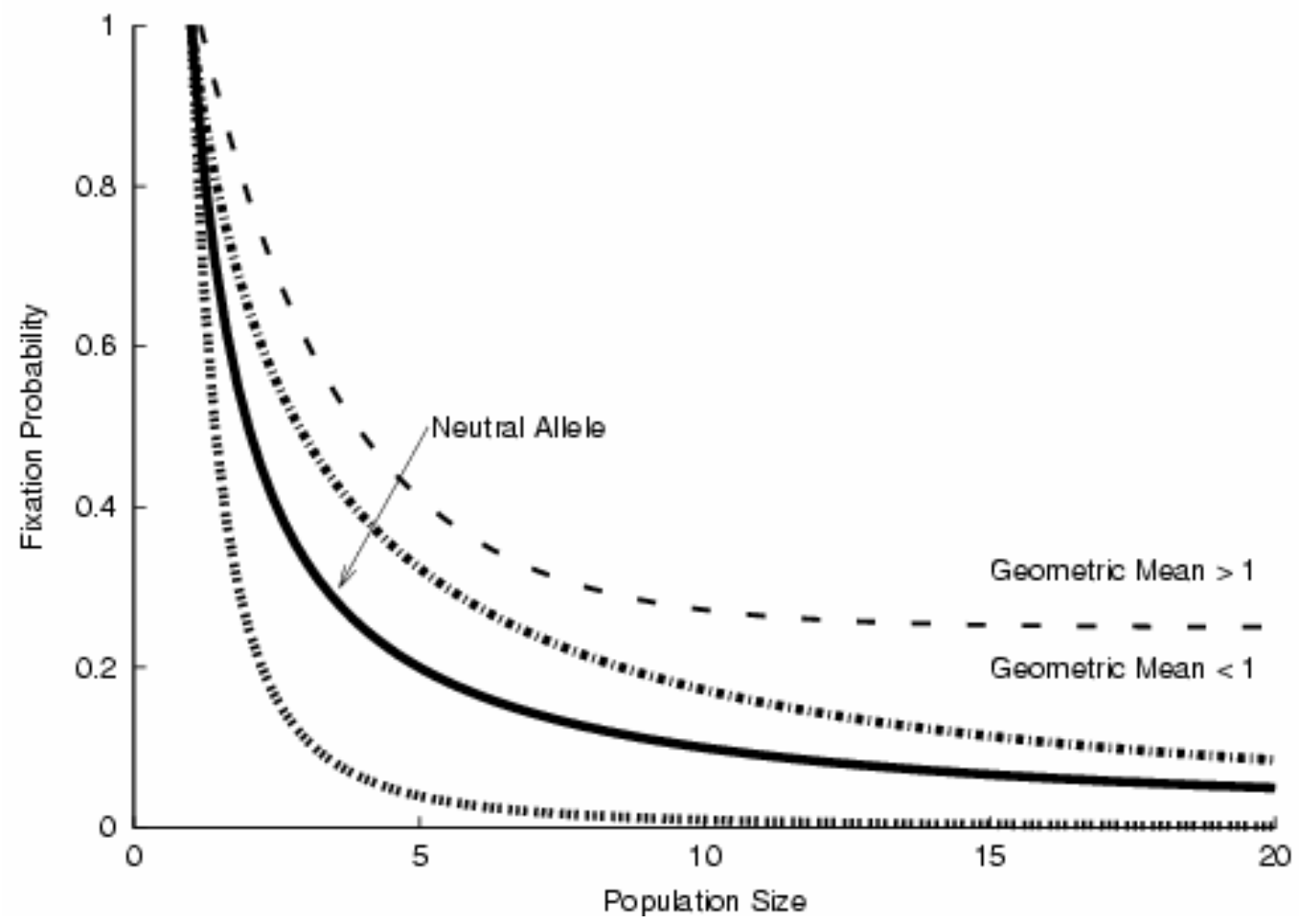

FIG. 2. This shows fixation probabilities as a function of population size for various invading alleles. The solid line is for a neutral allele, so the fixation probability is $1 / N$. The upper dashed line is for an allele which has a geometric mean greater than 1 . This fixation probability is above $1 / N$ and approaches a positive value as population size increases. The middle dashed curve is for an allele with a geometric mean less than 1, but a fixation probability greater than a neutral allele. The lower dashed curve is for an allele which has a geometric mean less than 1 and a fixation probability less than a neutral allele 
which will be observed more often in the longterm, regardless of population size. This is true even if both alleles have geometric mean fitnesses of less than 1 , as there is no reason why the ratio of the fixation probabilities cannot become more different as population size becomes large.

\subsection{Adaptive dynamics}

A second justification for using probability of fixation comes from a more sophisticated model of evolution that includes a range of possible alleles. This is precisely what Dieckmann and Law modeled to derive the gradient equations of adaptive dynamics, given a simplified model of fixation (Dieckmann and Law, 1996). In their framework, a branching process approximation is used to describe the dynamics of rare invaders, which leads to the calculation that maladapted alleles have a fixation probability of zero. However, the actual fixation probabilities could instead be used in their calculations, with an analogous result (appendix $B)$. Evolution will, on average, take the population up the fixation probability gradient. One interesting difference between these two approaches is that in the Dieckman and Law model, asymmetric mutation kernels (which can be thought of as a bias in the direction of mutation) slow down the approach to the attracting point, but do not alter the equilibrium state; in the fixation probability framework mutation bias causes the mean state to move away from the original attractor. This suggests that we might use the probability of fixation of strategy $x$ when rare against $y$ (i.e. $U(x, y))$ as a 'fitness function' in the standard analyses used in game-theoretic and adaptive dynamics modeling when the model of interest involves stochasticity. This allows potential generalizations of the traditional ESS definition, as well as definitions which include dynamic stability, like 'continuously stable strategies' (CSS) and the evolutionary attractors of adaptive dynamics (Maynard Smith, 1982; Eshel, 1983; Taylor, 1989; Christiansen, 1991; Geritz et al., 1998). When strategies exist against which all other strategies have less than neutral fixation probabilities then they represent the stochastic generalization of an ESS. If this strategy has higher than neutral fixation probabilities against all mutant strategies then it also invades all other strate- gies, and this can be thought of as a global evolutionary attractor. Similar arguments could be made to generate a stochastic analogue of the CSS condition of game theory and adaptive dynamics (which is a local condition).

Other more complex scenarios are also possible, for instance a set of strategies could be mutually invadible or uninvadible (Jensen, 1973; Karlin and Levikson, 1974; Yoshimura and Clark, 1991; Proulx, 2000). Even in these more complex scenarios the fixation probability gradient can be used to find the mean population state (appendix $B$ ), but the possibility of the stochastic version of evolutionary branching (Abrams et al., 1993; Metz et al., 1996a; Geritz et al., 1998) cannot be excluded.

It should be noted, however, that both of the above arguments assume that mutations are infrequent when compared to the time scale at which replacement occurs. However, if population size is large enough or the mutation rate is high many alternative alleles will segregate in the population at any point in time. In addition, two alleles could coexist for long periods of time or coexist as a polymorphism, putting the above arguments in doubt. Interactions between multiple alleles could also alter the dynamics of a focal allele, particularly if disruptive selection results in population splitting (Abrams et al., 1993; Geritz et al., 1998). While it is likely that these complications reduce the utility of fixation probability in some situations, the results discussed below show that fixation probability can still provide useful evolutionary information, even for these more complicated situations.

\section{Lottery competition in stochastic environments}

The above arguments for using the fixation probability of rare alleles to follow evolution under environmental stochasticity would have little weight if the results from doing so did not differ much from those that rely on the geometric mean. In this section we show that results using the geometric mean argument and those using the fixation probability differ in interesting ways for the lottery model with environmental stochasticity (Turelli, 1978b; Chesson and Warner, 1981; Real and Ellner, 1992; Kisdi and Meszena, 1995; Ranta et al., 
2000). Furthermore, simulation results suggest that up to reasonably large population sizes, the fixation probability results reflect the actual adaptive dynamics.

The lottery model is designed to mimic the life cycle of a sessile, iteroparous organism with fixed population size (Chesson and Warner, 1981). The population size is assumed to be limited by sites for which juveniles must compete, but can also be applied to species which consume a fixed amount of any resource which is renewed each generation. The order of events is zygote production $\rightarrow$ adult mortality $\rightarrow$ zygotes compete for available sites $\rightarrow$ zygote production. Mortality occurs independently in each site with a probability given by the strategy of the occupant of that site. Thus, the number of surviving adults of a particular strategy is binomial. The number of zygotes produced is assumed to be well in excess of the total number of sites, so that the number of sites which are filled by each strategy is also a binomial random variable. Many sessile marine organisms and plants fit this model well, and non-sessile organisms may fit it as well if some other resource has a fixed yearly production and individuals can consume at most some fixed amount of it.

Kisdi and Meszena (1995) have considered an example where stochasticity is introduced to the lottery model via mortality, and there is a trade-off between mean mortality and fecundity. In each year the mortality of adults is determined by multiplying a random environmental variable by the mortality probability of the strategy. The expected frequency in the next generation is

$$
\begin{array}{r}
p_{t+1}=p_{t} s_{m} \xi_{t}+\frac{p_{t} n_{m}}{p_{t} n_{m}+\left(1-p_{t}\right) n_{r}} \\
\left(1-p_{t} s_{m} \xi_{t}-\left(1-p_{t}\right) s_{r} \xi_{t}\right)
\end{array}
$$

where $p_{t}$ is the mutant frequency at generation $t, n$ represents the fecundity of a strategy, $s$ is the survivorship of a strategy, the subscripts $m$ and $r$ refer to mutant and resident, and $\xi_{t}$ is a stochastic environmental effect which alters survivorship. We assume a trade-off between fecundity and survivorship taken from Kisdi and Meszena (1995) so that

$$
s(n)=0.8-0.5\left(\frac{e^{n}}{10^{3}+e^{n}}+\frac{e^{0.6 n}}{10^{5}+e^{0.6 n}}\right)
$$

Thus, a mutant strategy will consist of a fecundity $n_{m}$ and an associated survivorship $s_{m}$ found by equation 3. Even if we only have one type of year (so that $\xi$ is fixed), the actual frequency in the next generation will vary around this mean, due to the stochastic nature of both death and replacement. However, if we take the limit as population size becomes large, then this variance is reduced to zero; only variance due to the environment will remain. Now consider the invasion criterion of the geometric mean growth rate of a mutant, when that mutant is rare. The growth rate when rare is found by taking the limit of $\frac{p_{t+1}}{p_{t}}$ as the frequency of the mutant allele goes to zero, and is

$$
\lambda_{m, r}=s_{m} \xi_{t}+\left(1-s_{r} \xi_{t}\right) \frac{n_{m}}{n_{r}}
$$

The expected $\log$ of $\lambda$ must be greater than zero for the rare strategy to increase. Figure 3 shows the pairwise invasibility plot for a constant environment and for a random environment (using the geometric mean of $\lambda$ ). The number of uninvadible strategies goes from 1 in a constant environment to 2 in a random environment.

The fixation probability of rare mutants may not be reflected by the geometric mean growth rate of a rare mutant. In this example, relative fixation probabilities can be easily found by examining the expected change in gene frequency (appendix $C$ ) using our original equation for the expected gene frequency in a finite population (equation 2). Because the random variable $\xi_{t}$ occurs only linearly, the expected frequency depends only on the arithmetic mean of $\xi_{t}$,

$$
\begin{aligned}
E\left[p_{t+1}\right]= & p_{t} s_{m} \bar{\xi}+ \\
& +\frac{p_{t} n_{m}}{p_{t} n_{m}+\left(1-p_{t}\right) n_{r}} \\
& \left(1-p_{t} s_{m} \bar{\xi}-\left(1-p_{t}\right) s_{r} \bar{\xi}\right),
\end{aligned}
$$



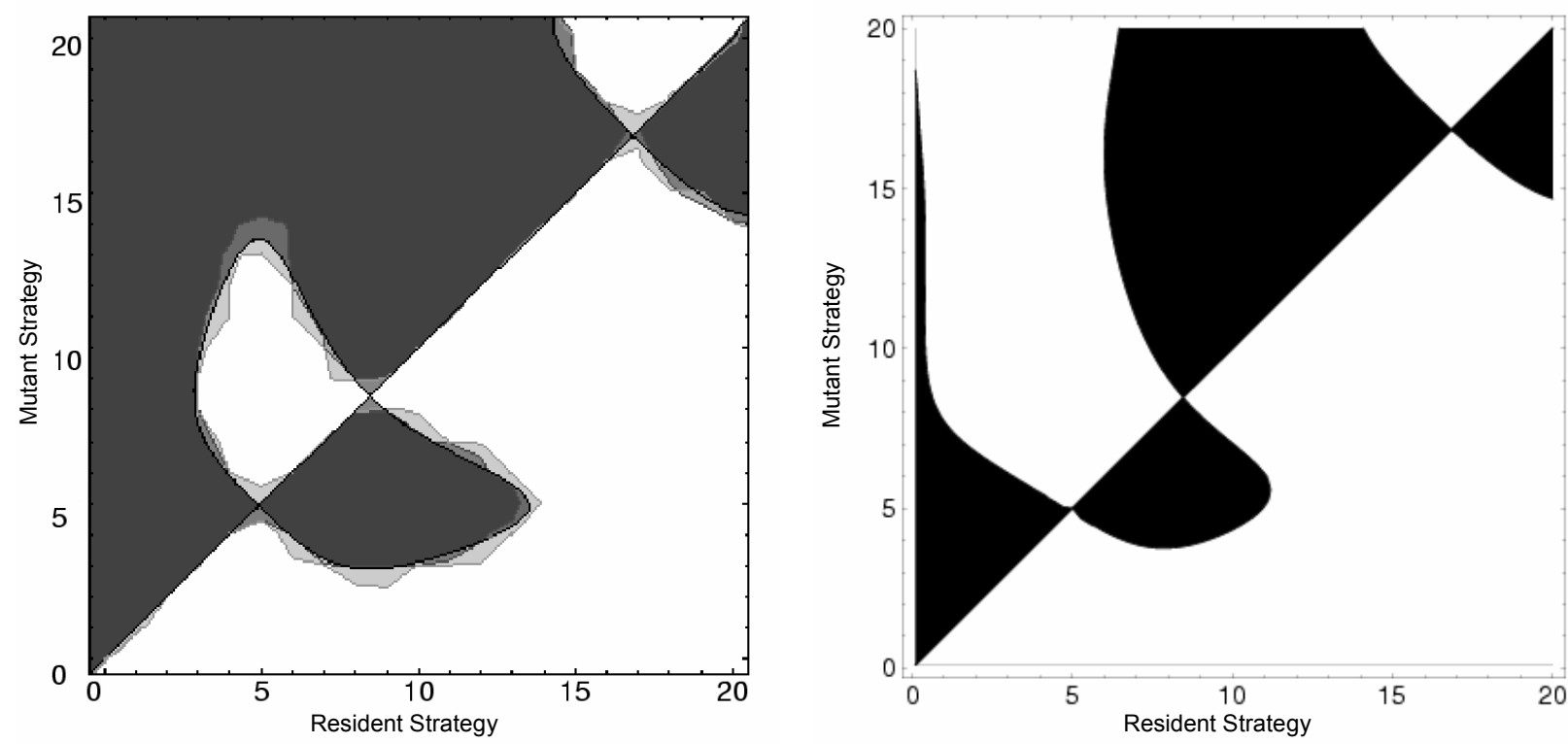

FIG. 3. Pairwise invasibility and replacement plots for the lottery model with stochastic death rates. The trade-off between birth and death rates taken from Kisdi and Meszena (1995) is $s(n)=0.8-0.5\left(\frac{e^{n}}{10^{3}+e^{n}}+\frac{e^{0.6 n}}{10^{5}+e^{0.6 n}}\right)$. The first panel shows the theoretical prediction, along with simulated fixation probabilities for populations of 500 individuals, for two models. The first simulated model has no yearly stochasticity in death rates, but is stochastic due to genetic drift (see text). The second simulated model includes stochasticity in death rates as well as genetic drift. The lightest shading is for drift only, while the medium shading is for both drift and stochasticity. There were 500 populations of 1,000 individuals for the drift simulation and 1,000 populations of 1,000 for the stochastic simulations. The shaded areas represent invasion/replacement of the strategy on the abscissa. The fixation probabilities for the stochastic model are predicted to be the same as for the deterministic model, which is borne out by their close association. The second panel shows the invasion criterion based on the geometric mean fitness when rare redrawn from Kisdi and Meszena (1995)

and the expected change in $p$ (i.e. $\left.E\left[p_{t+1}-p_{t}\right]\right)$ is

$$
\begin{aligned}
E[\Delta p]= & \frac{p_{t}\left(1-p_{t}\right)}{p_{t} n_{m}+\left(1-p_{t}\right) n_{r}} \\
& \left(n_{r}\left(s_{m} \bar{\xi}-1\right)-n_{m}\left(s_{r} \bar{\xi}-1\right)\right) .
\end{aligned}
$$

Note that the sign of the expected $\Delta p$ does not depend on $p_{t}$ or on population size. If we take the mean of $\xi$ to be 1, as in Kisdi and Meszena (1995), then we see that stochasticity does not qualitatively alter the pattern of fixation probabilities. That is, the same alleles which had higher than neutral fixation probabilities without stochasticity still have higher than neutral fixation probabilities with stochasticity. This is in direct contrast to findings based on the geometric mean, as shown in Figure 3. Also, the expected $\Delta p$ does not depend on population size, so this qualitative pattern of evolution is not altered by increasing population size. Large population size still plays a role, however, by making evolutionary transitions from one type to another less frequent. This is shown in Figure 4, where the actual fixation probabilities are shown. Even when the fixation probability is above $1 / N$, it may be quite small.

In one case studied by Kisdi and Meszena, the system has one ESS in a constant environment (ESS A) and, by the geometric mean argument, two ESS's in the stochastic environment (ESS A and ESS B, see Fig. 3). What happens is that stochasticity lowers $\lambda$ for ESS A when it is invading ESS B. This means that in an infinite population composed of individuals playing $\mathrm{B}$, a mutant playing A will eventually go extinct. However, in a finite population of any size it is possible for A to replace $\mathrm{B}$, and it is more likely than the converse. This means that if these two strategies were pitted against one another, the population would be found at $\mathrm{A}$ more of the time. Interestingly, these arguments are true regardless of the population size for the lottery model. If A and B were pitted against 


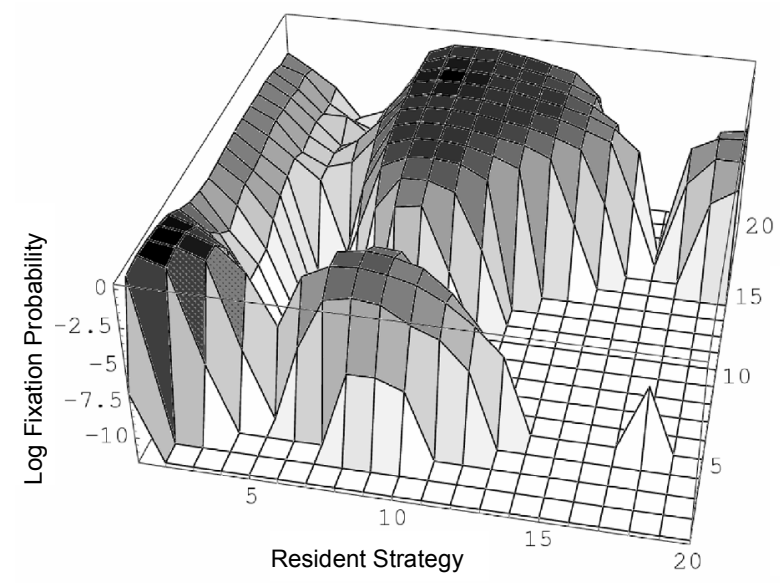

FIG. 4. This shows the $\log$ of fixation probabilities for the lottery model with stochastic death rates in a population of 500 individuals. The shading is based on the fixation probability, with all white panels representing fixation probabilities of less than $1 / 500$

one another in an extremely large population, then we would very rarely see any transitions from out of either state, but transitions from B to A would still be much more frequent than the reverse, indicating that the original ESS is still evolutionarily favored. The time required for these transitions becomes extremely large as population size increases however, so, while the system spends an increasing proportion of time fixed for $\mathrm{A}$, the time required for a transition from $\mathrm{B}$ to $\mathrm{A}$ may be so large as to be evolutionarily unimportant.

\subsection{Simulation results}

The analyses based on fixation probability depend on separating the time scales of mutation and replacement. If replacement takes long enough, or the mutation rate is high enough, then many alleles should segregate in the population at any point in time. Also, the time taken for replacement, given that it occurs, may depend on the invading allele. Therefore, we designed a simulation which allows for a continuum of alleles with mutation between alleles. In this simulation each genotype is associated with a fecundity and survivorship (a strategy). This model includes both environmental stochasticity and genetic drift. Genetic drift is included because this is an individual based model so only integer numbers of adults and offspring are allowed. Both the number of surviving adults and number of successful offspring are therefore modeled as binomial trials.

In this model, adults first produce gametes and then experience mortality. The number of gametes produced depends upon the genotype of the parent, and the gamete genotype is modified by mutation with a probability dependent on the mutation rate parameter. Mutation is assumed to operate globally, so that each genotype is equally likely to arise by mutation from any other genotype. We adopted this model of mutation because a strictly local mutational process would find local equilibria in both the stochastic and constant models, and it is only the global properties of the equilibria which are predicted to change under stochasticity. Each adult then dies with a probability determined by her

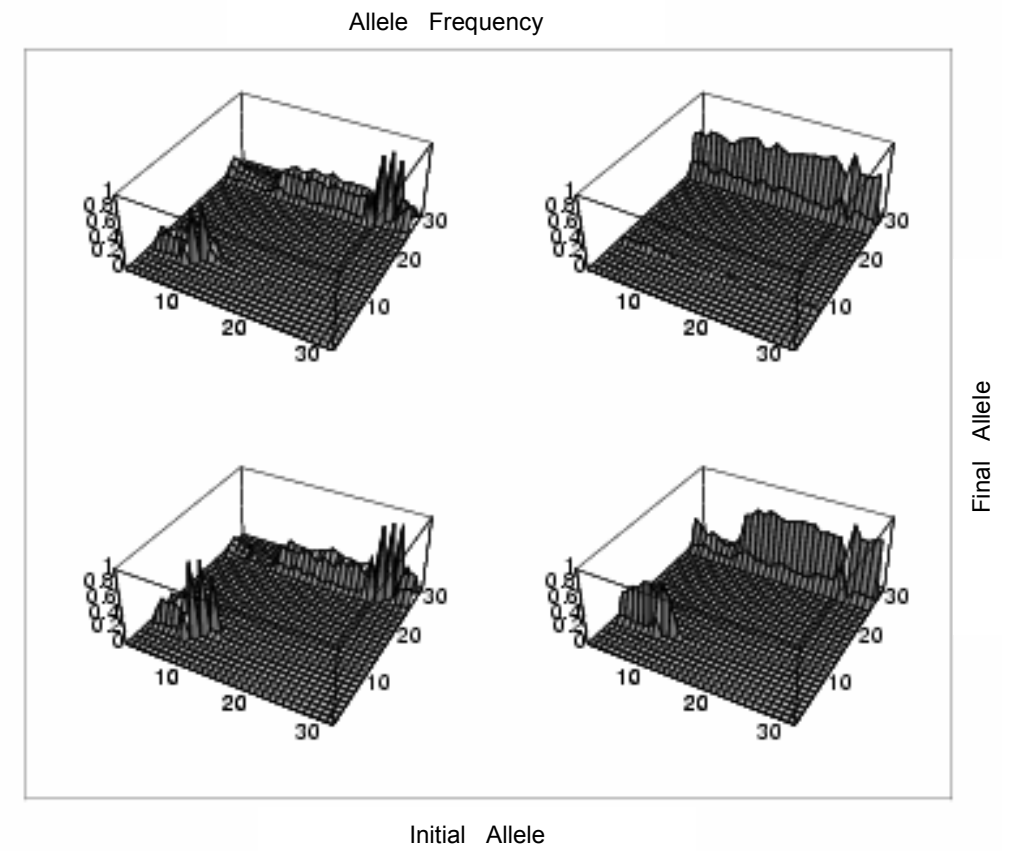

FIG. 5. This shows the dependence of the distribution on population size and time. The simulation was run with 30 alleles with offspring production between 0 and 20 and a mutation rate of 0.001 . The $x$-axis represents the initial population genotype, while the $y$-axis shows a frequency distribution averaged across 100 runs of the simulation. The top row is for populations of size 1,000 , while the bottom row is for populations of size 10,000 , while the bottom row is for populations of size 10,000 . The left column shows the distributions after only 1,000 generations and the right column shows the distribution after 10,000 generations 
genotype and the current environment, which is itself a random variable. The empty spots are then filled by juveniles which are drawn at random from the pool of gametes. At the beginning of each run all individuals had the same genotype. We ran the simulation 100 times for each set of conditions for two different lengths of time.

Figure 5 shows the gene frequency distribution, averaged over all the runs, for a given initial condition. After a long time, the set of populations should approach a stationary distribution, independent of the initial condition. This effect is demonstrated in the top half of Figure 5, for a modestly sized population of 1,000 individuals. After only 1,000 generations, the populations which started fixed for ESS B still have a high frequency of individuals around $\mathrm{B}$. However, by generation 10,000 , most individuals have genotype $\mathrm{A}$, regardless of the initial state of the population. These results are replicated for a larger population of 10,000 individuals in the lower half of the figure. Now, even after 10,000 generations there is still an effect of the initial condition, although it is less pronounced than at 1,000 generations.

\section{Conclusions}

We have argued that the probability of fixation provides important information for predicting the course of evolution in stochastic environments for finite populations. Although probability of fixation can sometimes be difficult to calculate analytically, the above example makes clear that using alternative measures (particularly those based on the geometric mean fitness) can easily give very different evolutionary predictions. Fixation probabilities can always be generated by simulation, and it is also possible in some contexts to generate analytical predictions about fixation probabilities relative to that of a neutral allele without calculating them explicitly (Proulx, 2000) (appendix C). Moreover, calculating the geometric mean fitness of a strategy (when rare or otherwise) can also be very difficult in all but the simplest of models (Metz et al., 1992; Ferriere and Gatto, 1995).

In order to present our argument for fixation probability as an indicator of evolutionary dynamics we adopted several simplifying assumptions. We limited our consideration to two segregating alleles in a population of fixed size and ignored potential differences in the time needed for replacement. These ideas can be easily extended to populations which fluctuate in size as long as population size is bounded both below and above. However, if extinction is possible, and particularly if the risk of total population extinction depends on the allele frequencies in the population then fixation probability will be poorly defined and unsatisfactory. Once the possibility of population extinction exists a more detailed examination of both population and genetic dynamics is required. Similarly, for some models, extensions to multiple segregating alleles are not difficult. The time dynamics were simplified by assuming that mutations occur with a frequency that is much less than the time that one allele takes to replace another. Under this assumption, the Markov chain or adaptive dynamics model does not depend on the time taken for allelic replacement.

If instead mutations occur often compared to the time required for alleles to fix, then the time dynamics of allele frequency become important. Asymmetries in fixation time could alter the expected evolutionary trajectory from the equilibrium predicted by fixation probability. This is a definite shortcoming of the probability of fixation as an indicator of evolutionary dynamics, which can only be resolved through models with increased complexity or simulation. We feel that the probability of fixation represents a medium where sensible predictions can be made, but where some realism is necessarily excluded.

Our simulations of lottery competition include many alleles and allow mutation to act at the same time scale as replacement. The simulations bear out most of our predictions: If the population size is not too large and the mutation rate is not too small, then the fixation probability gradient accurately predicts the mean population genotype. These simulations do reveal that as population size becomes large the time required to reach this state can be very large and may become evolutionarily unimportant. However, we do note that even the smaller population size of 1,000 individuals is reasonably large.

While the geometric mean has been used as an invasion criterion for some one dimensional stochastic systems, the Lyapunov exponent has been used as a measure of stability in multi-dimensional 
and one dimensional chaotic models (Metz et al., 1992; Ferriere and Gatto, 1995). In fact, the geometric mean of a rare strategy is the Lyapunov exponent for a one dimensional system. These more complex systems differ from the one analyzed in this paper in that the population dynamic attractors can be stable cycles or chaotic trajectories. Clearly, when Lyapunov exponent techniques are applied to stochastic systems, the issues raised here will still apply, but we believe the difficulty is even more severe. It seems quite plausible that even in deterministic systems with chaotic attractors the Lyapunov exponent can fail as an invasion measure. The Lyapunov exponent is calculated by assuming that the mutant trajectory stays infinitesimally close to the attractor for an arbitrarily long period of time (Rand et al., 1994), just as the geometric mean argument did for one dimensional stochastic systems. A negative dominant Lyapunov exponent can result from alternating increasing and decreasing mutant dynamics, so that periods of mutant growth can cause the trajectory to deviate from the attractor, and into a range where the linear approximation no longer applies. This will be particularly true in finite populations, because changes of even a single individual result in finite deviations from the attractor.

Our intent here is not to discredit the geometric mean as a measure of evolutionary change, but rather to point out that the utility of the geometric mean has not been fully quantified. Models with finite populations can yield substantially different results than models which use the geometric mean fitness of rare alleles, and the extent to which population size alters these results is an open question. Frequency dependence creates additional difficulties which the geometric mean, as a local measure, cannot overcome. The arguments presented here for the probability of fixation of rare alleles must be tempered by population dynamic and extinction effects of alternative alleles. Although the geometric mean may represent a suitable fitness measure for some population structures, there is still much theoretical work required before a complete understanding of evolution in even simple stochastic environments is achieved.

\section{Acknowledgements}

We would like to thank Eva Kisdi, Stefan Geritz, J. A. J. Metz, Peter Abrams and Steve Ellner for reading and making extensive comments on earlier drafts of this manuscript. Our thinking also benefited from conversations with Michael Doebeli, Fred Adler, Erik Noonburg and Jeremy Quastel. Funding was provided by NSERC and a Premiers Research Excellence Award to TD.

\section{APPENDIX}

\section{A) The geometric mean as a fitness measure}

The geometric mean as a fitness measure seems to have been first derived for a model of two clones, each experiencing independent growth. In this model, the growth of each clonal population is considered independently, so that the growth equations are

$$
N_{i}(t+1)=R_{i}(t) N_{i}(t),
$$

where $N_{i}(t)$ is the number of clone $i$ individuals at time $t$ and $R_{i}(t)$ is the number of type $i$ offspring produced per capita in generation $t$. The frequency of type $i$ is simply

$$
\begin{aligned}
p(t+1) & =\frac{N_{1}(t+1)}{N_{1}(t+1)+N_{2}(t+1)}= \\
& =\frac{N_{1}(t) R_{1}(t)}{N_{1}(t) R_{1}(t)+N_{2}(t) R_{2}(t)} .
\end{aligned}
$$

We are interested in how the frequency of each clone changes in the long run, so we would like a formula for $p(t)$ in terms of the starting frequency. By defining the selection coefficient as $s(t)=\frac{R_{2}(t)}{R_{1}(t)}$ and writing the $N_{i}$ 's in terms of $p$, we see that

$$
p(t)=\frac{p(0)}{p(0)+(1-p(0)) \prod_{t} s(t)} .
$$

So, it seems that the long run behavior of $p$ depends on the product of all the selection coefficients experienced, and does not depend on their order. What happens to the product of the selection coefficients after a long time? 


$$
\prod_{t} s(t)=\left(e^{\ln \left(\left(\prod_{t} s(t)\right)^{\frac{1}{t}}\right)}\right)^{t}=\left(e^{\sum_{t} \frac{\ln (s(t))}{t}}\right)^{t} .
$$

The final expression in equation 10 includes the sum of a random outcomes, weighted by how many times each outcome occurs. By the law of large numbers, the number of times each event occurs approaches the probability of that event times the number of events. The geometric mean $\left(M_{g}\right)$ of a random variable $X$ is defined as

$$
M_{g}=e^{\sum \ln (x) \operatorname{Pr}(X=x)},
$$

where $\operatorname{Pr}(X=x)$ is the probability that a particular value $x$ occurs, so equation 10 approaches

$$
\prod_{t} s(t)=\left(e^{\sum_{t}^{\ln (s(t)) \operatorname{Pr}(s(t))}}\right)^{t}=M_{g}^{t} .
$$

therefore, if $M_{g}>1$, then the product of selection coefficients grows without bound, but if $M_{g}<1$ the product of selection coefficients approaches 0 . In case where $M_{g}=1$, the product of selection coefficients approaches 1. Substituting this back into equation 9 we get

$$
p(t)=\frac{p(0)}{p(0)+(1-p(0)) M_{g}^{t}} .
$$

We know that $M_{g}^{t}$ will approach either 0 , 1, or infinity, and so $p(t)$ will either approach $1, p(0)$, or 0 , respectively.

\section{B) Deriving the adaptive dynamics equation}

The equations for adaptive change used in adaptive dynamics models have been derived by Dieckmann and Law (1996) by using a branching process approximation for the success of a rare mutant. They define the fixation probability of a rare mutant as the probability that the mutant does not go extinct when mutant birth and death rates are determined by the conditions set up by a resident population at equilibrium. In their derivation, the probability of invasion of a neutral mutant is exactly zero, and the probability of invasion of mutants with growth rates less than 1 is also zero. This results in a stochastic process which can only move in certain directions and cannot, even rarely, move down an adaptive landscape.

They first derive the transition probability for a population as the product of the probability a mutant enters the population and the probability of fixation. They have explicitly assumed that the timescales are separated, so that fixation occurs before subsequent mutation. This gives

$w\left(s^{\prime}, s\right)=\mu(s) b(s) \hat{n}(s) M\left(s, s^{\prime}-s\right) \frac{f\left(s^{\prime}, s\right)}{b\left(s^{\prime}, s\right)}$,

where $\mu(s)$ is the mutation rate, $b(s)$ is the birth rate, $\hat{n}(s)$ is the equilibrium population size, $M\left(s, s^{\prime}-s\right)$ is the probability of a mutational jump from $s$ to $s^{\prime}, f\left(s^{\prime}, s\right)$ is the net reproductive rate of the mutant, $s$ is the resident strategy, and $s^{\prime}$ is the mutant strategy. In their branching process derivation $\frac{f\left(s^{\prime}, s\right)}{b\left(s^{\prime}, s\right)}$ is the fixation probability of a mutant, so the equation could be rewritten as

$w\left(s^{\prime}, s\right)=\mu(s) b(s) \hat{n}(s) M\left(s, s^{\prime}-s\right) U\left(s^{\prime}, s\right)$,

where $U\left(s^{\prime}, s\right)$ is defined as the probability that $s^{\prime}$ reaches fixation from a single starting mutant in a population otherwise composed of $s$.

The mean path dynamics can be derived in similar ways for both formulations. The mean path, which is the average of population states over many runs of the process, is defined as

$$
\langle s\rangle(t)=\int s P(s, t) d s,
$$

where $P(s, t)$ is the probability density of genotype $s$ at time $t$. The dynamics of the mean path can be derived following Dieckmann and Law (1996). We will write $\langle s\rangle$ as $s$ to simplify the notation, giving

$$
\frac{d s(t)}{d t} \int_{R}\left(s^{\prime}-s\right) w\left(s^{\prime}, s\right) d s^{\prime},
$$


where $R$ is the set over which integration is performed. The range of integration is the set of mutations which have some chance of invading, so that $R=\left\{s^{\prime} \mid f\left(s^{\prime} ; \mathrm{s}\right)>0\right\}$ for the Dieckman and Law treatment, and $R$ is the entire domain for our treatment. We can now substitute in the two forms for $w$ and approximate the systems with a Taylor's series expansion around small $s^{\prime}-s$. For the Dieckman and Law treatment we have

$$
\frac{d s(t)}{d t}=\mu(s) \hat{n}(s) f^{\prime}(s) \int_{R}\left(s^{\prime}-s\right)^{2} M\left(s, s^{\prime}-s\right) d s^{\prime},
$$

and for our treatment we have

$$
\begin{aligned}
\frac{d s(t)}{d t}= & \mu(s) b(s)\left(\int_{R}\left(s^{\prime}-s\right) M\left(s, s^{\prime}-s\right) d s^{\prime}+\right. \\
& \left.+\hat{n}(s) U^{\prime}(s) \int\left(s^{\prime}-s\right)^{2} M\left(s, s^{\prime}-s\right) d s^{\prime}\right)
\end{aligned}
$$

Note that the range of integration is quite different in the two cases. In the Dieckman and Law formulation with a symmetric mutational distribution, only half of the local mutations (those which increase the fixation probability) have a non-zero chance of replacing the resident. In our formulation, all possible mutants have some non-zero chance of replacing the resident. We can define the proportion of mutants which increase fitness as $\rho(s)$.

If we define $\bar{M}=\int_{R}\left(s^{\prime}-s\right) M\left(s, s^{\prime}-s\right) d s^{\prime}$ and $\sigma^{2}=\int\left(s^{\prime}-s\right)^{2} M\left(s, s^{\prime}-s\right) d s^{\prime}$ then the two approaches, respectively, yield

$$
\frac{d s(t)}{d t}=\rho(s) \mu(s) \hat{n}(s) f^{\prime}(s) \sigma^{2},
$$

and

$$
\frac{d s(t)}{d t}=\mu(s) b(s)\left(\bar{M}+\hat{n}(s) U^{\prime}(s) \sigma^{2}\right) .
$$

Interestingly, the approach based on fixation probabilities shows some dependence on directional biases in the mutation function. In the Dieckman and Law formulation only the magni- tude of mutational steps is important, not the direction because deleterious mutations can never be fixed. In our formulation a directional bias in mutation will push the mean path away from the putative ESS, as would be expected in a quantitative genetic model.

\section{C) Fixation probability as a martingale}

If the mean change in frequency of a mutant allele has a constant sign, regardless of frequency, then it is easy to determine whether the fixation probability is larger or smaller than that of a neutral allele Proulx (2000). This type of stochastic process is called a submartingale when the initial value of the process remains above the expected value at every time in the future (it is called a supermartingale if the expected value increases in time). The idea is that if, for any frequency, an allele is expected to spread, then compounding this process through time also results in the alleles spread. Finally, because this is a discrete model with two absorbing states (loss or fixation of the mutant) the continual increase in the expected allele frequency must translate into a higher probability of fixation than the initial frequency. The converse argument can be made for alleles which are expected to decrease in frequency, but for alleles which vary in their tendency to spread no such simple calculation can be made.

Mathematically, this can be proved by considering the stochastic sequence of the number of mutant alleles, $N_{t}$, which has two absorbing states, 0 and $N$ (the total population size). The martingale property means that the expected number of mutant alleles can only increase. Because there are only two absorbing states, the probability that the process is not in an absorbing state decreases towards 0 as time becomes large. Putting these two facts together with the definition of fixation probability leads to the conclusion that the probability that the mutant allele becomes fixed is greater than that of a mutant allele,

$$
U_{i} \geq \frac{i}{N},
$$

where $U_{i}$ is the probability a mutant allele becomes fixed given a starting number of $i$. 


\section{References}

Abrams, P. A., Matsuda, H. and Harada, Y. (1993): Evolutionary unstable fitness maxima and stable fitness minima of continuous traits. Evol. Ecol. 7:465-487.

BENTON, T. B. and GRANT, A. (1999): Optimal reproductive effort in stochastic, density-dependent environments. Evolution 53:677-688.

Benton, T. B. and Grant, A. (2000): Evolutionary fitness in ecology: comparing measures of fitness in stochastic, density-dependent environments. Evol. Ecol. Res. 2:769-789.

Bulmer, M. G. (1985): Selection for iteroparity in a variable environment. Amer. Nat. 126:63-71.

Chesson, P. L. (1985): Coexistence of competitors in spatially and temporally varying environments: a look at the combined effects of different sorts of variability. Theor. Popul. Biol. 28:263-287.

Chesson, P. L. and Ellner, S. (1989): Invasibility and stochastic boundedness in monotonic competition models. $J$. Math. Biol. 27:117-138.

Chesson, P. L. and WARNeR, R. R. (1981): Environmental variability promotes coexistence in lottery competitive systems. Amer. Natur. 117:923-943.

Christiansen, F. B. (1991): On conditions for evolutionary stability for a continuously varying character. Amer. Natur. 138:37-50.

Crow, J. F. and Kimura, M. (1970): An Introduction to Population Genetics Theory. Harper and Row, New York.

DieCKMANN, U. and LAW, R. (1996): The dynamical theory of coevolution: a derivation from stochastic ecological processes. J. Math. Biol. 34:579-612.

ELLNER, S. (1984): Asymptotic behavior of some stochastic difference equation population models. J. Math. Biol. 19:169-200.

ELLNER, S. P. (1996): You bet your life: Life-history strategies in fluctuating environments, In OTHMER, H. G., ,ADLER, H. G., Lewis, M. A. and DALlon, J. C. (eds), Case Studies in Mathematical Modeling: Ecology, Physiology and Cell Biology. Prentice Hall, Upper Saddle River, New Jersey, pp. 3-24.

EsHEL, I. (1983): Evolutionary and continuous stability. $J$. Theor. Biol. 103:99-112.

FERRIERE, R. and GATtO, M. (1995): Lyapunov exponents and the mathematics of invasion in oscillatory or chaotic populations. Theor. Popul. Biol. 48:126-171.

Geritz, S. A. H., Kisdi, E., Meszena, G. and Metz, J. A. J. (1998): Evolutionarily singular strategies and the adaptive growth and branching of the evolutionary tree. Evol. Ecol. 12:35-57.

GiLLESPIE, J. H. (1973): Natural selection with varying selection coefficients - a haploid model. Genet. Res. 21:115120.

Gillespie, J. H. (1974): Natural selection for within generation variance in offspring number. Genetics 76:601-606.

GRANT, A. (1997): Selection pressures on vital rates in density-dependent populations. Proc. R. Soc. Lond. B 264: 303-306.
HACCOU, P. and IwASA, Y. (1996): Establishment probability in fluctuating environments: a branching process model. Theor. Popul. Biol. 50:254-280.

Heino, M., Metz, J. A. J. and Kaitala, V. (1998a): The enigma of frequency-dependent selection. Trends Ecol. Evol. 13:367-370.

Heino, M., Metz, J. A. J. and Kaitala, V. (1998b): Reply. Trends Ecol. Evol. 13:509.

IwASA, Y. (1988): Free fitness that always increases in evolution. J. Theor. Biol. 135:265-281.

IwASA, Y. and LEVIN, S. A. (1995): The timing of life history events. J. Theor. Biol. 172:33-42.

JENSEN, L. (1973): Random selective advantages of genes and their probabilities of fixation. Genet. Res. 21:215-219.

KARLIN, S. and LEVIKSON, B. (1974): Temporal fluctuations in selection intensities: case of small population size. Theor. Popul. Biol. 6:383-412.

KISDI, E. (1998): Frequency dependence versus optimization. Trends Ecol. Evol. 13:508.

KisDi, E. and MeszenA, G. (1995): Life histories with lottery competition in a stochastic environment: ESSs which do not prevail. Theor. Popul. Biol. 47:191-211.

MAYNARD SMith, J. (1982): Evolution and the Theory of Games. Cambridge University Press, Cambridge.

Metz, J. A. J., Geritz, S. A. H., Meszena, G., Jacobs, F. J. A. and VAn HEerwaARDEN, J. S. (1996a): Adaptive dynamics, a geometrical study of the consequences of nearly faithful reproduction. In STREIN, S. J. VAN and VERDuYn Lunel, S. M. (eds), Stochastic and Spatial Structures of Dynamical Systems. North Holland, Amsterdam, pp. 183-213.

Metz, J. A. J., Mylius, S. D. and Diekmann, O. (1996b): When does evolution optimise? On the relation between types of density dependence and evolutionary stable life history parameters. Technical report, IIASA Working Papers WP-96-004.

Metz, J. A. J., Nisbet, R. M. and Geritz, S. A. H. (1992): How should we define 'fitness' for general ecological scenarios? TREE 7:198-202.

Отто, S. P. and WhiтLOCK, M. C. (1997): The probability of fixation in populations of changing size. Genetics 146: 723-733.

ProulX, S. R. (2000): The ESS under spatial variation with applications to sex allocation. Theor. Popul. Biol. 58:3347.

Rand, D. A., Wilson, H. B. and McGlade, J. M. (1994): Dynamics and evolution: evolutionarily stable attractors, invasion exponents and phenotype dynamics. Philos. Trans. R. Soc. Lond. B 343:261-283.

Ranta, E., Tesar, D., Alaja, S. and Kaitala, V. (2000): Does evolution of iteroparous and semelparous reproduction call for spatially structured systems? Evolution 54:145-150.

Real, L. A. and Ellner, S. (1992): Life history evolution in stochastic environments: A graphical approach. Ecology 73:1227-1236. 
Seger, J. and Brockmann, H. J. (1987): What is bethedging? In Oxford Surveys in Evolutionary Biology, Volume 4, pp. 182-211.

TAYLOR, P. D. (1989): Evolutionary stability in one-parameter models under weak selection. Theor. Popul. Biol. 36:125143.

TuRELLI, M. (1978a): Does environmental variability limit niche overlap? Proc. Natl. Acad. Sci. 75:5085-5089.
TuRELLI, M. (1978b): A reexamination of stability in randomly varying versus deterministic environments with comments on the stochastic theory of limiting similarity. Theor. Popul. Biol. 13:244-267.

Yoshimura, J. and Clark, C. W. (1991): Individual adaptations in stochastic environments. Evol. Ecol. 5:173-192. 
